
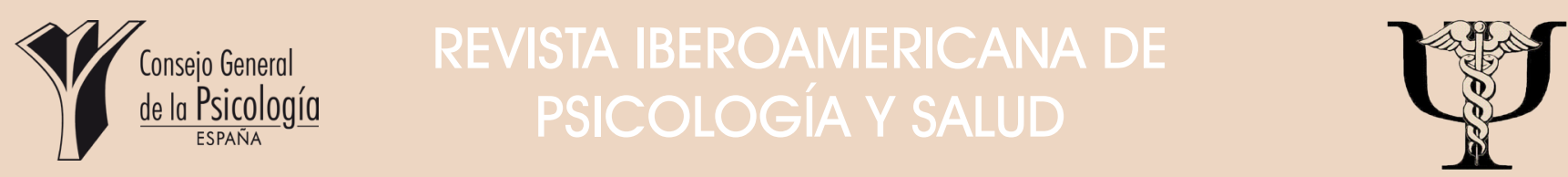

Revista Oficial de la Federación Iberoamericana de Asociaciones de Psicología (FIAP) [Official Journal of the Latin-American Federation of Psychological Associations]

\title{
Actitud sexista y trascendente durante el noviazgo entre universitarios latinoamericanos.
}

\section{Carolina Bringas-Molleda', Cristina Estrada-Pineda ${ }^{2}$, Javier Suárez-Álvarez ${ }^{3}$ Andrea Torres ${ }^{3}$, Francisco Javier Rodríguez-Díaz ${ }^{3 *}$, Eduardo García-Cueto ${ }^{3}$ y Luis Rodríguez-Franco ${ }^{4}$}

'Dpto. Psicología y Antropología. Universidad de Extremadura (España)

${ }^{2}$ Dpto. Trabajo Social. Universidad de Guadalajara (México)

${ }^{3}$ Dpto. Psicología. Universidad de Oviedo (España)

${ }^{4}$ Dpto. Personalidad, Evaluación y Tratamientos Psicológicos. Universidad de Sevilla (España)

- Recibido: 4 - Agosto - 2016 - Aceptado: 26 - Noviembre - 2016

\section{RESUMEN}

Las actitudes de rol de género ante situaciones de diversos ámbitos, contribuyen significativamente a nuestra forma de actuar, y a la aceptación o rechazo de conductas que consideramos o no pertinentes en un momento dado. Nuestro objetivo es examinar el desarrollo de las actitudes de rol de género en las relaciones de noviazgo de estudiantes universitarios de México y Argentina mediante la adaptación de la escala EARG / GRAS. Participaron un total de 2013 estudiantes universitarios de ambos sexos con edades comprendidas entre 17 y 26 años. Se les pasó el Cuestionario de Violencia entre novios (CUVINO), la escala de actitudes de rol de género (EARG) y el cuestionario de personalidad EPQ-RA. Los resultados mostraron una estructura bidimensional en la escala de actitudes de género en ambos países (actitudes trascendentes y sexistas), que se relacionan significativamente con los factores de violencia y tolerancia al maltrato. Asimismo, las mujeres se muestran significativamente sexistas, y en México hallamos una mayor proporción en ambos tipos de actitud. También hallamos que a medida que aumenta la edad, incrementa la actitud sexista; una relación significativa entre una actitud igualitaria y el neuroticismo, y entre actitudes sexistas con psicoticismo y conformidad.

PALABRAS CLAVE: Actitudes, Edad, Sexo, Personalidad, Violencia.

\section{ABSTRACT}

Sexist and transcendent attitude during courtship between latin American university. The gender-role attitudes in situations, concerning various fields, contribute significantly to our behavior, and acceptance or rejection of behaviors that we consider relevant or not at any given time. Our aim is to examine the development of gender-role attitudes in dating relationships of university students in Mexico and Argentina by adapting the GRAS/ EARG scale. They involved a total of 2013 college students of both sexes aged between 17 and 26 years. They were provided with the questionnaire dating violence (CUVINO), the scale of gender-role attitudes (GRAS) and personality questionnaire EPQ-RA. The results show a two-dimensional structure on the scale of gender attitudes in both countries (transcendent and sexist attitudes), which correlated significantly with the factors of violence and abuse tolerance. Also, women are significantly sexist, and as for Mexico stands out for a larger share in both types of attitude. Moreover, results also exhibit that as age increases, growths the sexist attitude; a significant relation between an egalitarian attitude with neuroticism and psychoticism, and between sexist attitudes and compliance.

Los estudios realizados sobre la violencia de pareja informan de tasas de ésta contra las mujeres de entre un 10\% y 40\% (Bapat y Tracey,

*Correspondencia: Francisco Javier Rodríguez Díaz.

C.D: 33003, Universidad de Oviedo, España.

E-mail:gallego@uniovi.es

(C) 2017 Sociedad Universitaria de Investigación en Psicología y Salud. Publicado por Consejo General de Colegios Oficiales de Psicólogos, España. Este es un artículo Open Access
bajo la CC BY-NC-ND licencia (http://creativecommons.org/licencias/by-nc-nd/4.0/).
2012). De igual modo, las mujeres entre 16 y 24 años tienen mayor riesgo de abuso por un compañero íntimo (Amar y Gennaro, 2005), siendo la violencia en una relación de noviazgo un importante precursor de la violencia doméstica o violencia sexual en la pareja (Ureña, Romero, Casas, Viejo, y Ortega-Ruiz, 2015). Por ello, la violencia en las relaciones afectivas 
adolescentes se considera un problema grave de salud pública (Farhat, Haynie, SummersettRinggold, Brooks-Russell, ylannotti,2015; Shorey et al., 2015), conductual y social (Bonomi et al., 2012; Martin, Houston, Mmari, y Decker, 2012; Temple, Shorey, Tortolero, Wolfe, y Stuart, 2013), observándose que diferencias individuales pueden mediar el futuro de las relaciones afectivas (Bapat y Tracey, 2012;StepteauWatson, 2014). La intervención al respecto ha alcanzado positivos (Ruiz-Hernández, GarcíaJiménez, Llor-Esteban, y Godoy-Fernández, 2015). No obstante, es necesario identificar los factores de riesgo personales y contextuales del violencia en las relaciones entre jóvenes, dado que la adolescencia constituye un período de desarrollo en el que se adquieren creencias, actitudes y valores de género que pueden derivar en conductas violentas en las relaciones afectivas, especialmente si se acompaña de factores tales como la disciplina negativa de los padres o de factores de riesgo en la comunidad (Fuente y Herrero-Olaizola, 2012; Novo, Herbón, y Amado, 2016). En este sentido, se ha constatado que la flexibilidad en el desempeño del rol de género puede servir para fortalecer las relaciones hombre-mujer, pero simultáneamente también puede crear tensión en esas relaciones (Stepteau-Watson, 2014), lo que resulta fundamental para el desarrollo eficaz de la prevención e intervención (Arce, Fariña, y Novo, 2014).Por otro lado, ciertos rasgos de personalidad de los agresores fueron referidos como responsables de una situación de maltrato (Mapayi Boladale et al., 2015). En una revisión, Yu, Geddes y Fazel (2012) concluyeron que algunos trastornos de personalidad se asociaban con conductas antisociales y reincidencia en el maltrato. Dowgwillo, Ménard, Krueger y Pincus (2016) encontraron que los rasgos de personalidad patológica se asocian con la violencia realizada tanto por varones como por mujeres.

La aceptación o el rechazo de conductas que se consideran socialmente competentes - adecuadas en las relaciones de pareja (Arce, Fariña, y Vázquez, 2011), ha sido insuficientemente investigada, probablemente como resultado de la recopilación de información y datos en un solo momento temporal, lo que ha hecho difícil precisar qué variables son correlatos, precursores o resultados de la violencia de pareja (Ureña, Romero, Casas, Viejo, y OrtegaRuiz, 2015) y ha llevado consigo la necesidad de completar la información con la proporcionada por el contexto en el que se produce violencia en el noviazgo. A este respecto, se ha documentado que las actitudes precursoras de la violencia en las relaciones afectivas, cobrando especial relevancia durante el desarrollo del rol de género, esto es, creencias, opiniones que favorecen y, a veces, pretenden justificar la discriminación de las personas en función del sexo, especialmente entre adolescentes y jóvenes (Contreras y Cano, 2016). En relación a estos últimos, las tasas de prevalencia parecen similares para chicos y chicas, si bien las chicas presentan mayor probabilidad de experimentar violencia física grave, sexual, lesiones físicas, y miedo (BringasMolleda et al., 2015).

En concreto, se ha descubierto que existen diferencias en las experiencias y actitudes hacia la violencia en las relaciones afectivas del noviazgo tanto por género como por niveles de aculturación (DuPont-Reyes, Fry, Rickert, y Davidson, 2015; East, y Hokoda, 2015; Rueda, Williams, y Nagoshi, 2015). Sucintamente, los hombres aceptan con mayor facilidad ciertas actitudes hacia la violencia, suelen ser más tradicionales en sus actitudes de rol sexual, lo que les facilita implicarse en mayor número y variedad de experiencias como perpetradores de violencia en las relaciones interpersonales. En relación a las mujeres, estas constatan mayores niveles de agresión verbal (González-Guarda, Cummings, Pino, y Malhotra,2014; StepteauWatson, 2014). En esta línea, el estudio de López-Cepero, Rodríguez-Franco, RodríguezDíaz, y Bringas (2013) muestra los varones con mayor puntuación en actitudes sexistas respuesta evaluativa en razón de pertenecer a un sexo, observándose en ello intención de comportamiento discriminatorio-, en tanto que las mujeres destacan por poseer ideas más trascendentes-niegan las diferencias entre hombres y mujeres en cuanto a estatus y poder, enfrentándose y negando la estructuración y jerarquización social como clave de la adaptación. Además, también observaron los jóvenes con actitudes igualitarias perciben mayores indicadores de victimización.

La condición de trascendencia o igualitarismo, o por el contrario, de sexismo, es uno de los factores que repercuten en 
la percepción de maltrato, ligado ello a la formación de prejuicios y estereotipos, como el deseo de mantener la relación afectiva o la consideración de comportamientos agresivos en las relaciones interpersonales afectivas de pareja como normales. Ello definirá la formación de una mayor o menor tolerancia ante situaciones violentas por parte de alguno de los miembros de la pareja (García-Cueto et al., 2015; LópezCepero Borrego, Rodríguez-Franco, RodríguezDíaz, Bringas, y Paíno, 2015; Vega-Gea, Ortega-Ruiz,y Sánchez, 2016).

En relación a la cultura latina, esta se caracteriza por ser tradicionalmente patriarcal, lo que implica que los roles sean por un lado hombres fuertes que proporcionen el sustento a la familia, mientras que las mujeres se dedicarían a la crianza y serían sumisas y abnegadas (Bridges, Karlsson, y Lindly, 2015). La visión tradicional de los roles de género lleva a propiciar factores de riesgo en las relaciones afectivas de noviazgo, llegando a ser estas insatisfactorias cuando se desafían los roles (Ulloa, Jaycox, Skinner, y Osburn, 2008), siendo una parte de la pareja víctima. De este modo, los factores protectores de especificidad cultural se pueden perder con la aculturación, lo que conduciría a un aumento de satisfacción en las relaciones afectivas de noviazgo (Bowen et al., 2013).

La situación descrita ha llevado a elaborar múltiplesescalas para evaluar las actitudes relacionadas con el rol de género, destacando: Gender Role Conflict Scale(GRCS) de O'Neil, Helms, Gable, David, y Wrightsman(1986), Hostility Towards Women Scales(HTWS) de Check, Malamuth, Elias, y Barton (1985) y la Illinois Rape Myth Acceptance Scale(IRMAS) de Payne, Lonsway, y Fitzgerald (1999). Estas escalas fueron diseñadas para evaluar las actitudes unidireccionalmente, esto es, de los hombres hacia las mujeres, destacando la última de ellas por valorar las actitudes ante agresiones de tipo sexual.

Este estadio de la cuestiónhace necesario disponer de escalas útiles para evaluar las actitudes de rol de género tanto en hombres como mujeres e identificar actitudes sexistas e igualitarias o trascendentes. En esta línea destaca la Escala de Actitudes de Rol de Género,(EARG/ GRAS) de García-Cueto et al.(2015) y Social Rol Questionnaire (SRQ-R) de Babery Tucker (2006). La primera ofrece un único factor con dos dimensiones (trascendente y sexista), considerando tres áreas de interpretación de los resultados: Familiar, Social y Laboral.Por su parte, el SRQ-R evalúa dos factores que coinciden con las actitudes trascendentes y sexistas.

Así, al asumir que las actitudes pueden ser diferentes según el rol que hombres y mujeres desempeñan en la sociedad, se configura un contexto donde la violencia de género es considerada algo habitual o normalizado, no existiendo sensibilización ante ella. Ello nos lleva a plantear la realización de este estudio, para así explorar el desarrollo de las actitudes de rol de género en las relaciones de noviazgo en población universitaria en países de América Latina (México y Argentina), mediante la adaptación de la escala EARG / GRAS.

\section{MÉTODO}

\section{- PARTICIPANTES}

Participaron en el estudio 2013 estudiantes universitarios, procedentes de México $(N=$ $1540,76.5 \%)$ y Argentina ( $N=473,23.5 \%)$, con edades comprendidas entre los 17 y 26 años $(M=20.45, D T=2.06)$ resaltando que el principal agrupamiento en México se da entre los $19-20$ años $(N=802,52.1 \%)$ y en la muestra Argentina entre los 21-26 años $(N=328,69.3 \%)$. Por lo que respecta al sexo, 790 (39.2\%, siendo el $85.4 \%$ de México) son varones, en tanto que $60.8 \%$ restante $(N=1223$, siendo el $70.7 \%$ de México) son mujeres.

\section{- PROCEDIMIENTO E INSTRUMENTOS DE EVALUACIÓN UTILIZADOS}

Los estudiantes que participaron en el estudio fueron seleccionados de forma no probabilística en los diversos centros universitarios, siendo el único criterio de inclusión el haber mantenido una relación de pareja de al menos un mes de duración. En las instrucciones se les explicó el objetivo de la investigación, así como la forma de responder a las diferentes pruebas que se les suministraba. Al mismo tiempo, se aseguró el anonimato de los participantes y se destacó la importancia de que sus respuestas fueran sinceras. Se procedió a resolver cuantas dudas planteaban los participantes, tanto antes como durante el tiempo que dedicaban a responder 
las diferentes escalas. Se les facilitó, en primer lugar, un cuestionario creado ad hoc que recogía información sociodemográfica de los participantes, tales como edad, sexo, nivel de estudios, curso escolar, así como percepción de clase social o desempeño laboral.

Seguidamente, cumplimentaron el Cuestionario de Violencia entre Novios (CUVINO), de Rodríguez-Franco et al. (2010). Este consta de 42 ítems que evalúan comportamientos abusivos que pueden tener lugar en las relaciones afectivas de pareja. El formato de respuesta es una escala tipo Likert de cinco alternativas (que va de 0: Nunca hasta 4: Casi siempre). Adicionalmente, los participantes respondieron a cada uno de los ítems en función del nivel de molestia o malestar que tales conductas le ocasionan, a través de una escala tipo Likert. Los 42 ítems, en ambos casos (victimización y molestia), se agrupan en ocho factores: Desapego, Humillación, Sexual, Coerción, Físico, Género, Castigo Emocional e Instrumental. En este estudio, la fiabilidad de las dimensiones, alpha de Crombach, para el nivel de victimización osciló entre .92 para Humillación y 80 para Coerción; siendo para el total del instrumento de .94.La fiabilidad de los mismos factores en molestiaoscilóentre.88para Castigo Emocional y Desapego y.97 para el factor Físico; siendo de .99 para el total de la escala.

Se aplicó la Escala de Actitudes de Rol de Género (EARG/GRAS), de García-Cueto et al. (2015). Se compone de 20 ítems sobre actitudes relacionadas con el papel asignado a hombres y mujeres en la sociedad, facilitando la identificación de conductas agresivas en las relaciones afectivas. Cada ítem dispone de cinco alternativas de respuesta (desde 1: Muy en desacuerdo hasta 5: Muy de acuerdo), estando agrupadas en Actitudes trascendentes o igualitarias, y actitudes sexistas, que ofrecen en la prueba original una consistencia factorial de .99.

Finalmente, se aplicó la adaptación española (lbañez, Orted, Moro, Ávila, y Parcet (1999) del cuestionario de personalidad EPQRA de Eysenck y Eysenck que, a través de 24 ítems dicotómicos (Sí/No), evalúa tres factores de personalidad: Extraversión, Neuroticismo y Psicoticismo. Además, incluye una escala de Disimulo/Conformidad. Los índices de consistencia interna en el estudio original fueron de $.83, .77, .62$ y .81 , mientras que en nuestro estudio han sido .82, .88, .80 y .76, respectivamente.

\section{- ANÁLISIS DE DATOS}

En primer lugar, se ejecutó un Análisis Factorial Confirmatorio (AFC) con objeto de someter a prueba una estructura unidimensional del EARG/GRAS en las muestras mexicanas y argentinas de población universitaria. Además, se realizó un Análisis Factorial Exploratorio (AFE) para determinar la estructura factorial que mejor representara los datos en ambos países, utilizándose el método de Implementación Óptima de Análisis Paralelos propuesto por Timmerman y Lorenzo-Seva (2011), llevándose a cabo 10.000 remuestreos. Se utilizó la correlación policórica como matriz de entrada de los datos y el método de factorización utilizado fue el de mínimos cuadrados no ponderados y el método de rotación, Promin (Ferrando y Lorenzo-Seva, 2014).En relación al ajuste de los modelos, la literatura sugiere que es adecuado cuando el CFI $>.90$, y el RMSEA $<.08$ (Kline, 2011).

Para comprobar la invarianza factorial entre los grupos (México y Argentina), se realizaron varios $\mathrm{AFC}$ en el marco de los modelos de ecuaciones estructurales. En primer lugar, se comprobó el supuesto de invarianza configuracional, para acreditar si los mismos ítems pesaban en los mismos factores en ambos grupos. Para ello se realizó un AFC en cada grupo separadamente (Abad, Olea, Ponsoda, y García, 2011 ; Muthén y Muthén, 2009, 2010). A continuación, se realizaron varios modelos anidados de Análisis Factorial Confirmatorio Multigrupo para estudiar la invarianza de los pesos factoriales (i.e. Modelo 1), la invarianza de los umbrales (i.e. Modelo 2), y el supuesto de que las varianzas de los errores y las varianzas de los factores son iguales entre los grupos (i.e. Modelo 3), de acuerdo con los modelos propuestos con Muthén y Asparouhov (2002), y Muthén y Muthén (2010). Se utilizó el método de estimación Mínimos Cuadrados Ponderados robusto (WLSMV) indicado para datos ordenados categóricamente (Elosua, 2010; Koh y Zumbo, 2008; Muthén y Asparouhov, 2002). 
La evaluación progresiva de la invarianza factorial se ha realizado en función de los criterios siguientes: diferencia de chi-cuadrado utilizando la corrección DIFFTEST (Muthén y Muthén, 2010), diferencia en los valores del Índice de Ajuste Comparativo de Bentler (CFI; Cheung y Rensvold, 2002), solapamiento entre los intervalos de confianza de los errores cuadráticos medios de aproximación (RMSEA; Byrne, 2008; Elosua y Muñiz, 2010).

Con el fin de estudiar los índices de discriminación de los ítems se utilizó la correlación parcial ítem-test, eliminando el influjo del ítem. La estimación de la fiabilidad se realizó mediante el coeficiente alfa de Cronbach para datos ordinales (Elosua y Zumbo, 2008). El estudio de las evidencias de validez en relación con otras variables se realizó mediante el coeficiente de correlación de Pearson entre las dos subescalas resultantes del EARG/ GRAS -transcendente y sexista-y los rasgos de personalidad del EPQRA. También se estudió la relación entre la actitud trascendente y sexista y las dimensiones evaluadas mediante el Cuestionario de Violencia de Novios (CUVINO;Rodríguez-Francoet al., 2010).

Finalmente, se realizó un Análisis Multivariado de la Varianza(MANOVA) para estudiar las diferencias en la tipología actitudinal del EARG/GRAS (i.e. trascendente y sexista) en función del sexo, el país de procedencia y la edad de los participantes. La variable edad se agrupó en adolescentes (17-18 años: mexicanos $=272,17.7 \%$ y argentinos $=45$, 9.5\%), jóvenes (19-20 años:mexicanos $=802$, $52.1 \%$ y argentinos $=100,21.1 \%$ ) y adultos jóvenes (21-26 años: mexicanos $=466,30.3 \%$ y argentinos $=328,69.3 \%)$.Para decidir la significación estadística se utilizó un nivel de confianza del 95\%. La estimación del tamaño del efecto se realizó mediante la prueba de $x^{2}$ parcial. Los datos fueron analizados mediante el SPSS 19, el MPLUS 7, y el FACTOR 9.2 (LorenzoSeva y Ferrando, 2006).

\section{RESULTADOS}

\section{- DIMENSIONALIDAD DEL EARG/GRAS EN MÉXICO Y ARGENTINA}

El AFC muestra un deficiente ajuste de los datos a la estructura unidimensional obtenida en población española tanto para los participantes del ámbito universitario de Argentina $\left(x^{2} / g /=3.1\right.$, $\mathrm{CFI}=.72$, SRMR $=.067)$ como de México $\left(x^{2} / g l=21.79, \quad C F I=.57, \quad S R M R=.116\right)$.Los resultados del AFE muestran que la estructura factorial que mejor representa los datos es la bidimensional tanto para Argentina $\left(x^{2} / g /=2.78\right.$, $\mathrm{GFI}=.91, \mathrm{RMSR}=.060)$ como para México $\left(x^{2}\right)$ $g /=2.32, \mathrm{GFI}=.99, \mathrm{RMSR}=.040)$. En ambos casos, el estadístico de Bartlett $(p<.001)$ y el test de Kaiser-Meyer-Olkin (KMO > .70) muestran una buena adecuación de los datos para llevar a cabo el análisis factorial. En función del contenido de los ítems los factores fueron definidos como actitud trascendente y actitud sexista; la correlación entre ambos factores fue de -.635 para Argentina y -.392 para México utilizando el método de rotación Promin.

\section{- EVALUACIÓN PROGRESIVA DE LA INVARIANZA FACTORIAL}

La evaluación progresiva de la invarianza factorial ofrece en la Tabla 1 los índices de ajuste del AFC, realizado en cada grupo separadamente. En el caso de Argentina, el valor de $x^{2} / g /$ es 6.43, el CFI superior a .95 y el RMSEA inferior a .08. En el caso de México, el valor de $x^{2} / g$ les inferior a 5 , el CFI es cercano a .90 y el RMSEA inferior a .08. Si bien el ajuste es mejor en Argentina, en ambos grupos se podría aceptar un adecuado ajuste de los datos a la estructura factorial propuesta; siendo sostenible que, a pesar de tener un índice CFI más bajo en México que en Argentina, habida cuenta que el resto de valores, mantener el supuesto de invarianza configuracional.

Tabla 7

Análisis Factorial Confirmatorio para cada grupo

\begin{tabular}{|l|c|c|c|c|}
\hline Modelo & $\boldsymbol{x}^{2}$ (gl) & $\boldsymbol{x}^{2}$ (gl) & CFI & RMSEA(IC) \\
\hline Argentina & 1086.70 (169) & 6.43 & .96 & .059 (.056-.063) \\
México & 572.11 (169) & 3.38 & .89 & .071 (.065-.077) \\
\hline $\begin{array}{l}\text { Nota. } x^{2}=\text { Estadístico chi-cuadrado utilizando el método de estimación de } \\
\text { mínimos cuadrados ponderados robusto; gl = grados de libertad; CFI = Índice } \\
\text { de Ajuste Comparativo; RMSEA = Raíz Cuadrática Media de los Errores de }\end{array}$ \\
Aproximación; IC = Intervalo de Confianza.
\end{tabular}

El análisis progresivo de la invarianza factorial comienza planteando un modelo de AFC multigrupo sin restricciones (Modelo 0) sobre el que se irán anidando el resto de 
modelos. Como se puede observar en la Tabla 2, los índices de ajuste del Modelo 0 muestran un ajuste adecuado según los criterios previamente establecidos. A continuación, para comprobar el supuesto de invarianza métrica se propone un modelo anidado en el que los pesos factoriales son invariantes entre los grupos (Modelo 1). Sin bien la diferencia de chi-cuadrado es estadísticamente significativa, la diferencia entre los CFI de los dos modelos es inferior a .01 $(C F I m o d 1-\bmod 2=.001)$ y no existen diferencias estadísticamente significativas ( $\mathrm{NC}=90 \%$ ) entre los RMSEA de ambos modelos. En función de estos criterios, parece sensato aceptar el supuesto de invarianza métrica (Modelo 1), ya que en cualquier caso las diferencias estadísticamente significativas obtenidas deben interpretarse con bastante cautela (Kline, 2011).

Tabla 2

Análisis progresivo de la invarianza factorial mediante Análisis Factorial Confirmatorio Multigrupo.

\begin{tabular}{|c|c|c|c|c|}
\hline Modelo & $x^{2}(g l)$ & $\Delta x^{2}(g l)$ & CFI & RMSEA(IC) \\
\hline 0. Modelo base & \multicolumn{2}{|l|}{$1892.75(378)$} & .946 & $.063(.060,-.066)$ \\
\hline $\begin{array}{l}\text { 1. Invarianza } \\
\text { de las cargas } \\
\text { factoriales }\end{array}$ & $1871.67(396)$ & $\begin{array}{c}87.48(18) \\
p<.001^{*}\end{array}$ & .947 & .061 (.058,-.064) \\
\hline $\begin{array}{l}\text { 2. Invarianza } \\
\text { de las cargas } \\
\text { factoriales y los } \\
\text { umbrales }\end{array}$ & $1967.54(414)$ & $\begin{array}{c}78.29(18) \\
p<.001^{*}\end{array}$ & .944 & $.061(.058,-.064)$ \\
\hline $\begin{array}{c}\text { 3. Invarianza } \\
\text { de las cargas } \\
\text { factoriales, los } \\
\text { umbrales, y las } \\
\text { varianzas de error } \\
\text { y varianzas de los } \\
\text { factores }\end{array}$ & $2013.44(434)$ & $\begin{array}{c}119.65 \\
(20) \\
p<.001 *\end{array}$ & .943 & $.060(.057,-.063)$ \\
\hline \multicolumn{5}{|c|}{$\begin{array}{l}\text { Nota. } x^{2}=\text { Estadístico chi-cuadrado utilizando el método de estimación } \\
\text { de mínimos cuadrados ponderados robusto; gl = grados de libertad; } \\
\Delta x^{2}=\text { Diferencia chi-cuadrado utilizando la corrección DIFFTEST; CFI = Índice } \\
\text { de Aiuste Comparativo; RMSEA = Raíz Cuadrática Media de los Errores de } \\
\text { Aproximación; IC = Intervalo de Confianza. *El modelo de invarianza factorial } \\
\text { sería rechazado. }\end{array}$} \\
\hline
\end{tabular}

El siguiente modelo anidado (Modelo 2) comprueba la invariancia en las estructuras de medición y estima el umbral para cada indicador. Por esta razón, el modelo establece los umbrales como parámetros fijos. Como se puede observar en la Tabla 2, no hay diferencias estadísticamente significativas (NC = 90\%) en los RMSEA de ambos modelos (Modelo 1 - Modelo 2) y la diferencia entre los índices comparativos de Bentler son inferiores a .01 (CFlmod1-mod2 $=.003)$. En función de estos criterios parece sensato aceptar la invarianza de los pesos factoriales y los umbrales a través de los grupos.

A continuación, la escala de los factores es fijada a ser igual a través de los grupos (Modelo 3). Esto implica que las cargas factoriales, los umbrales, y las varianzas residuales son invariantes a través de los grupos. Como se puede observar en la Tabla 2: la diferencia entre los CFI de ambos modelos es inferior a .01 (CFlmod2-mod3 $=.001)$ y no hay diferencias estadísticamente significativas (NC $=90 \%$ ) entre los RMSEA de ambos modelos.

El coeficiente Alfa de Cronbach para el factor de actitudes trascendentes fue de .83 en Argentina y de .94 en México. En el caso del factor de actitudes sexistas, el coeficiente de fiabilidad fue de .85 para Argentina y de .89 para México. Los índices de discriminación en el factor de actitudes trascendentes oscilaron entre .28 y .78 para México y entre .20 y .40 para Argentina, mientras que en el factor de actitudes sexistas oscilaron entre .35 y .61 para México y entre .26 y .54 para Argentina.

\section{- EVIDENCIAS DE VALIDEZ EN RELACIÓN CON OTRAS VARIABLES}

Por lo que respecta a la correlación existente entre las dimensiones del EARG/GRAS (i.e. actitud trascendente y actitud sexista) y EPQ-RA (i.e. Extraversión, Neuroticismo y Psicoticismo), los coeficientes de correlación de Pearson muestran que los únicos índices estadísticamente significativos, aunque bajos, se encontraron entre la actitud trascendente y neuroticismo $(r=-.054, p<.05)$ y actitud sexista y psicoticismo $(r=-.110, p<.01)$.

Los resultados de validez convergente con las dimensiones comportamentales del CUVINO mostraron correlaciones negativas entre casi todos los factores conductuales y las actitudes sexistas. Sin embargo, al contrario de lo esperado, las correlaciones fueron negativas también con las actitudes igualitarias ante el maltrato por desapego, humillación, género y castigo emocional (ver Tabla 3).Por otro lado, los resultados muestran correlaciones negativas entre las dimensiones de molestia del CUVINO (i.e. desapego, humillación, sexual, coerción, físico, género, castigo emocional e instrumental) con el factor actitud sexista y correlaciones positivas con el factor actitud trascendente. La 
correlación más baja se encontró entre actitud sexista y molestia por desapego $(r=-.071, p$ $=.001)$ y la más alta entre actitud sexista y molestia sexual $(r=-.142, p<.01)$. Si bien las correlaciones son bajas, si se encuentran en la dirección de lo esperado (ver Tabla 4). del efecto es prácticamente de cero; aunque el tamaño del efecto también es muy pequeño en el caso de la Actitud Sexista, en este caso las diferencias sí son estadísticamente significativas $(p<.001)$. La prueba de Bonferroni, utilizada para las comparaciones post hoc muestra que existen diferencias entre todos los grupos de edad $(p<.01)$ excepto entre jóvenes y adolescentes $(p=.407)$.

Correlaciones entre las dimensiones del EARG/GRAS y los factores conductuales del CUVINO

\begin{tabular}{|c|c|c|c|c|c|c|c|c|}
\hline & DE & HU & SE & co & FI & GE & CA & IN \\
\hline $\begin{array}{l}\text { Actitud } \\
\text { trascendente }\end{array}$ & $-.049^{\star}$ & $-.049^{*}$ & .006 & $.074^{\star *}$ & $.091^{\star *}$ & $-.090^{\star \star}$ & -.042 & .033 \\
\hline $\begin{array}{l}\text { Actitud } \\
\text { sexista }\end{array}$ & $-.072^{\star \star}$ & $-.053^{\star}$ & $-.109 * *$ & $-.157^{\star \star}$ & $-.135^{\star \star}$ & -.034 & $-.128^{\star \star}$ & $-.126^{\star *}$ \\
\hline
\end{tabular}

Tabla 4

Correlaciones entre las dimensiones del EARG/GRAS y los factores de tolerancia del CUVINO

\begin{tabular}{|c|c|c|c|c|c|c|c|c|}
\hline & DE & HU & SE & CO & $\mathbf{F I}$ & GE & CA & IN \\
\hline $\begin{array}{l}\text { Actitud } \\
\text { trascendente }\end{array}$ & $.071^{* *}$ & $.077^{* *}$ & $.091^{* *}$ & $.054^{*}$ & $.089 * *$ & $.071^{* *}$ & $.074^{\star *}$ & $.092^{\star \star}$ \\
\hline $\begin{array}{l}\text { Actitud } \\
\text { sexista }\end{array}$ & $-.071^{* *}$ & $-.078^{* *}$ & $-.142^{\star *}$ & $-.073^{\star \star}$ & $-.084^{\star \star}$ & $-.108^{\star *}$ & $-.076^{* *}$ & $-.086^{* *}$ \\
\hline
\end{tabular}

Tabla 5

Diferencia de medias en Actitud trascendente y sexista en función del sexo, país y edad.

\begin{tabular}{|c|c|c|c|c|c|c|c|c|c|c|c|c|c|}
\hline \multicolumn{3}{|c|}{ Sexo } & \multicolumn{5}{|c|}{ País } & \multicolumn{6}{|c|}{ Edad } \\
\hline & H & $M$ & $p$ & $\eta^{2}$ & $M_{x}$ & Ar & $p$ & $\eta^{2}$ & $17-18$ & $19-20$ & $21-26$ & $p$ & $\eta^{2}$ \\
\hline AS & 41.88 & 50.37 & $<.001$ & .24 & 47.39 & 44.86 & $<.001$ & .02 & 45.23 & 45.95 & 47.19 & $<.001$ & .01 \\
\hline
\end{tabular}

Nota. $\mathrm{AT}=$ Actitud Trascendente; $\mathrm{AS}=$ Actitud Sexista; $\mathrm{H}=$ Hombres; $\mathrm{M}=$ Mujeres; $\eta 2=$ Tamaño del efecto Eta Cuadrado Parcial; $M x=$ México; $\mathrm{Ar}=\mathrm{Argentina}$.

\section{- ESTUDIO DE LAS DIFERENCIAS EN FUNCIÓN DEL SEXO, EL PAÍS Y LA EDAD}

Los resultados tal y como aparecen en la Tabla 5 muestran que existen diferencias estadísticamente significativas entre hombres y mujeres, tanto en actitud transcendente como sexista $(p<.001)$; hay que señalar que el tamaño del efecto es bajo en actitud transcendente $\left(x^{2} .20\right)$ y medio en la Actitud Sexista $\left(x^{2}>.20\right)$. Respecto a los países también aparecen diferencias estadísticamente significativas entre Argentina y México tanto en Actitud Transcendente como en Actitud Sexista $(p<.001)$, si bien en ambos casos el tamaño del efecto es bajo $\left(x^{2}<.20\right)$. En cuanto a la edad, no existen diferencias estadísticamente significativas en Actitud Transcendente $(p=.19)$ y además el tamaño

\section{DISCUSIÓN}

Las creencias y actitudes pueden determinar en gran medida la formación de roles de género, que juegan un papel importante en la definición de las funciones y responsabilidades que las personas desempeñan en la sociedad. Cuando estas creencias y actitudes están basadas en estereotipos erróneos pueden dar lugar a situaciones indeseables dentro de la relación afectiva de pareja, como conductas violentas (García-Cueto et al., 2015), o un alto nivel de tolerancia ante potenciales situaciones de abuso (Rodríguez, Antuña, López-Cepero, Rodríguez, y Bringas, 2012).En suma, parece sensato desarrollar instrumentos de medida, desde la 
perspectiva de género, para evaluar las actitudes relacionadas con el rol de género, ya que tendría implicaciones en la modificación y prevención de conductas violentas en relaciones de pareja. En esta línea, el objetivo de investigación es adaptar la Escala de Actitudes de Rol de Género (GarcíaCueto et al., 2015) en México y Argentina, así como explorar la relación de las actitudes de rol de género en las relaciones de pareja a nivel transcultural.

La comparación transcultural de las actitudes y roles de género requirió una serie de requisitos. En primer lugar, se realizó un análisis factorial confirmatorio para comprobar la estructura factorial del EARG/GRAS en México y Argentina. Los resultados mostraron un deficiente ajuste de los datos en ambos países a la estructura unifactorial obtenida previamente en población española. A continuación, se realizó un análisis factorial exploratorio con el objetivo de identificar la estructura factorial, encontrándose la solución bifactorial como la mejor alternativa para ambos países. Los dos factores fueron definidos como actitud trascendente y actitud sexista, de acuerdo a las dimensiones del modelo unifactorial de GarcíaCueto et al. (2015); la correlación entre ambos factores fue de - .635 para Argentina y -.392 para México. Estos resultados sugieren que el papel de las actitudes de rol de género no sea el mismo en España que en México y Argentina, lo que concuerda con quienes afirman que las culturas y contextos reflejan diferentes perspectivas en la asignación de funciones concretas a varones y mujeres (East, y Hokoda, 2015; Fariña, Arce, y Novo, 2008; Marco, Gracia, Tomás, y LópezQuilez, 2015).

Por otro lado, la evaluación progresiva de la invarianza factorial permite confirmar la asunción fuerte de invarianza métrica ente México y Argentina. Esto implica que no sólo las cargas factoriales y los umbrales son invariantes a través de ambos grupos, sino que las varianzas de error y las varianzas de los factores son también invariantes. En otras palabras, el constructo es medido con niveles similares de precisión en ambos grupos (Dimitrov, 2010). En suma, se confirma que si bien el instrumento original validado en población española muestra una estructura esencialmente unidimensional (García-Cueto et al., 2015), en el caso de México y Argentina la estructura que mejor se ajusta a los datos en la población universitaria participante es la bidimensional.

En cuanto a las relaciones entre las actitudes de rol y las dimensiones de personalidad, los resultados muestran índices de correlaciones negativos significativos entre las actitudes trascendentes y neuroticismo y entre las actitudes sexistas y dureza emocional. Si bien el sexismo se caracteriza portratar de justificar la discriminación basada en las diferencias de género, podría también relacionarse en cierta medida con una personalidad egocéntrica y caracterizada por la dureza emocional. Sin embargo, es necesario explicar la relación positiva y significativa con una actitud igualitaria, sustentada en que no refiere ningún concepto de desigualdad en relación al género y la estabilidad emocional, caracterizado por emociones negativas. En cierta medida, los datos se interpretarían en dos sentidos. De un lado, las relaciones, aunque estadísticamente significativas, son de una magnitud pequeña y de modo tentativo se podrían inferir que las actitudes transcendentes se encuentran ligadas a una mayor sensibilidad personal, mientras que las sexistas más estrechamente vinculadas la insensibilidad social. Por otro lado, las diferencias a nivel de género pueden referirse en torno a la dureza emocional, interpretando que los varones suelen adscribirse con ello al rol de agresores y las mujeres al de víctima (Fawson, 2015; Herrero, Torres, Fernández-Suárez, y Rodríguez-Díaz, 2016).

Uno de los aspectos más importantes, que explican el interés de este estudio es la correlación obtenida entre la percepción de victimización y las creencias sobre el papel social desempeñado por ambos sexos. Quienes muestran una actitud caracterizada por el establecimiento de relaciones igualitarias entre hombres y mujeres informan de mayores indicadores de victimización, debido a que reconocen cuando aparece una situación de abuso dentro de la pareja (Cortés-Ayala et al, 2015;López-CeperoBorrego et al., 2015). En la población de nuestro estudio, México y Argentina, se refleja que aquellos jóvenes que mantenían actitudes basadas en el sexismo son quienes referían significativamente menores indicadores de victimización en todos los tipos de abuso, a excepción del maltrato de género, donde la correlación se sitúa en la misma dirección, aunque sin ser significativa. Esto se explica por la dificultad en el reconocimiento 
de éste, considerándolo normalizado en la relación de pareja (Cortés-Ayala et al., 2015). Sin embargo, los datos no están tan claros cuando nos referimos a las actitudes trascendentes o igualitarias, pues encontramos cuatro factores de violencia que correlacionan negativamente con un mayor igualitarismo. Es probable que siesta población se caracteriza por la tendencia protectora de los varones y de subordinación femenina (Ulloa et al., 2008), no esté tan claro el concepto igualitario de asignación de responsabilidad que ambos miembros de la pareja tienen en la sociedad, y dentro de la propia relación afectiva. Sin embargo, al evaluar el nivel de tolerancia mediante el grado de molestia que proporcionan las conductas de maltrato, se ha constatado que el igualitarismo correlaciona positivamente con el grado de molestia ante la posible ocurrencia de todas las situaciones de maltrato. En la misma línea, una actitud sexista correlaciona negativamente tanto con el nivel de malestar que le ocasiona la situación de maltrato como con la probabilidad de que ocurriera.

Si bien ha quedado clara la existencia de dos factores de la escala del EARG/GRAS en la población universitaria mexicana y argentina, al realizar el análisis diferencial por las variables sociodemográficas de sexo, país y edad, llama la atención que sean las mujeres quienes se reivindiquen como más sexistas. Esto puede explicarse por las características propias del contexto cultural que asigna un papel dominante al varón. Adicionalmente, es previsible que las respuestas de los varones estén sesgadas por disimulación, es decir, tendencia a ocultar características negativas (Arce, Fariña, Seijo, y Novo, 2015).Si diferenciamos por países, México se caracteriza por una mayor proporción de personas con actitudes tanto trascendentes como sexistas. En cuanto a la edad, hallamos que, a mayor edad, más actitudes y conductas sexistas. Teniendo en mente que la población del estudio es de un nivel educativo superior, de los resultados se infiere que la cultura incide, en este contexto, en un concepto de superioridad del varón frente a la mujer, de lo que se deriva que el varón asume un rol de dominancia en la relación (Bowen et al., 2013; García-Cueto et al., 2015; López-
Cepero Borrego et al., 2015; Ulloa et al., 2008).

En relación a las limitaciones de este estudio, resalta que los resultados están basados en auto informes con las implicaciones que esto conlleva como la percepción subjetiva de la realidad vivida o la honestidad en las respuestas; en este caso, disimulación. Por esto, para un abordaje más profundo sería recomendable replicar estos estudios con un diseño longitudinal de las parejas adolescentes, ahondando en las conductas agresivas tanto de los hombres como las mujeres. Además, el diseño del presente estudio no aborda la estabilidad de las variables de medida en el sujeto inter-contextos.

\section{- Conflicto de intereses}

Los autores declaran no tener ningún conflicto de intereses.

\section{- Agradecimientos}

Esta investigación fue financiada con proyectos del Ministerio de Sanidad, Política Social e Igualdad (SUBMINMU012/009) y de la AECID_CYTED(AP/035718/11).

\section{REFERENCIAS}

Abad, F.J., Olea, J., Ponsoda, V., y García, C. (2011). Medición en ciencias sociales y de la salud. Madrid: Síntesis.

Amar, A.F., y Gennaro, S. (2005). Dating violence in college women: Associated physical injury, healthcare usage, and mental health symptoms. Nursing Research, 54(4), 235-242.

Arce, R., Fariña, F., y Novo, M. (2014). Competencia cognitiva en penados primarios y reincidentes: Implicaciones para la reeducación [Cognitive competence among recidivist and non-recidivist prisoners: Implications for the rehabilitation]. Anales de Psicología, 30(1), 259-266. http://dx.doi. org/10.6018/analesps.30.1.158201

Arce, R., Fariña, F., Seijo, D. y Novo, M. (2015). Assessing impression management with the MMPI-2 in child custody litigation. Assessment, 22(6), 769-777. http://dx.doi.org/10.1177/1073191114558111

Arce, R., Fariña, F., y Vázquez, M. J. (2011). Grado de competencia social y comportamientos antisociales delictivos y no delictivos en menores [Social competence and delinquent, antisocial, and non-deviant behavior in adolescents]. Revista Latinoamericana de Psicología, 43(3), 473-486. Recuperado de: http://publicaciones. konradlorenz.edu.co/index.php/rlpsi/article/ view/487/583 
Baber, K.M.,y Tucker, C.J. (2006). The social roles questionnaire: A new approach to measure attitudes toward gender. Sex Roles, 54, 459-467. http://dx.doi. org/10.1007/s $11199-006-9018-y$

Bapat, M., y Tracey, T.J.G. (2012). Coping with dating violence as a function of violence frequency and solution attribution: A structural modeling approach. Violence and Victims, 27(2), 329-343

Bonomi, A.E., Anderson, M.L., Nemeth, J., BartleHaring, S., Buettner, C., y Schipper, D. (2012). Dating violence victimization across the teen years: Abuse frequency, number of abusive partners, and age at fist occurrence. BMC Public Health, 12(1), 637-646. http://dx.doi.org/10.1186/1471-2458-12-637

Bowen, E., Holdsworth, E., Leen, E., Sorbring, E., Helsing, B., Jaans, S., y Awouters, V. (2013). Northern European adolescent attitudes toward dating violence. Violence and Victims, 28(4), 619-634. http:// dx.doi.org/10.1891/0886-6708.W-D-12-0009

Bridges, A.J., Karlsson, M., y Lindly, E. (2015). The effect of brief, passive psychoeducation on knowledge and ratings of intimate partner violence in the United States and Argentina. Journal of Interpersona/ Violence, 30(2), 272-294. http://dx.doi. org/10.1177/0886260514534775

Bringas-Molleda, C., Cortés-Ayala, L., FloresGalaz, M., Antuña-Bellerín, A., López-Cepero, J., y Rodríguez-Díaz, F.J. (2015). Análisis diferencial de la percepción de jóvenes sobre maltrato en el noviazgo. Revista Latinoamericana de Ciencias Sociales, Niñez y Juventud, 13(2), 477-488.

Byrne, B.M (2008).Testing for multigroup equivalence of a measuring instrument:A walk through the process. Psicothema, 20(4), 872-882.

Check, J.V.P., Malamuth, N.M., Elias, B., y Barton, S.A. (1985). On hostile ground: Do you have feelings of hostility toward the opposite sex? Psychology Today, April, 56-61.

Cheung, G.W., y Rensvold, R.B. (2002).Evaluating goodness-of-fit indexes for testing measurement invariance. Structural Equation Modeling, 9, 233-255. http://dx.doi.org/10.1207/S15328007SEM0902 5

Contreras, L., y Cano, M.C. (2016). Child-to-parent violence: The role of exposure to violence and its relationship to social-cognitive processing. European Journal of Psychology Applied to Legal Context, 8(2), 4350. http://dx.doi.org/10.1016/i.eipal.2016.03.003

Cortés-Ayala, L., Flores, M., Bringas, C., RodríguezFranco, L., López-Cepero, J., y Rodríguez, F. (2015). Relación de maltrato en el noviazgo de jóvenes mexicanos. Análisis diferencial por sexo y nivel de estudios. Terapia Psicológica, 33(1), 5-12.
Dimitrov, D. M. (2010). Testing for factorial invariance in the context of construct validation. Measurement and Evaluation in Counseling and Development, 43(2), 121-149. http://dx.doi. org/10.1177/0748175610373459

Dowgwillo, E.A., Ménard, K.S., Krueger, R.F., y Pincus, A.L. (2016). DSM-5 Pathological personality traits and intimate partner violence among male and female college students. Violence and Victims, 37(3), 416437. http://dx.doi.org/10.1891/0886-6708.W-D-1400109

DuPont-Reyes, M., Fry, D., Rickert, V., y Davidson, L.L. (2015). Adolescent relationship violence and acculturation among NYC Latinos. Maternal and Child Health Journal, 19(7), 1543-1552. http://dx.doi. org/10.1007/s10995-014-1659-9

East, P.L., y Hokoda, A. (2015). Risk and protective factors for sexual and dating violence victimization: A longitudinal, prospective study of Latino and African American adolescents. Journal of Youth and Adolescence, 44(6). 1288-1300. http://dx.doi. org/10.1007/s10964-015-0273-5

Elosua, P., y Muñiz, J. (2010).Exploring the factorial structure of the self-concept. European Psychologist, 15(1), 58-67. http://dx.doi.org/10.1027/1016-9040/ $\underline{\mathrm{a} 000006}$

Elosua, P., y Zumbo, B. (2008). Coeficientes de fiabilidad para escalas de respuesta categórica ordenada. Psicothema, 20(4), 896-901.

Farhat, T., Haynie, D., Summersett-Ringgold, F., Brooks-Russell, A., y lannotti, R.J. (2015). Weight perceptions, misperceptions, and dating violence victimization among U.S. adolescents. Journal of Interpersonal Violence, 30(9), 1511-1532. http:// dx.doi.org/10.1177/0886260514540804

Fariña, F., Arce, R., y Novo, M. (2008). Neighborhood and community factors: Effects on deviant behavior and social competence. The Spanish Journal of Psychology, 17(1), 78-84. http://dx.doi.org/10.1017/ $\underline{S 1138741600004133}$

Fawson, P.R. (2015). Controlling behaviors as a predictor of partner violence among heterosexual female and male adolescents. Partner Abuse, 6(2), 217-229. http://dx.doi.org/10.1891/1946-6560.6.2.217

Ferrando, P.J., y Lorenzo-Seva, U. (2014). El análisis factorial exploratorio de los ítems: Algunas consideraciones adicionales. Anales de Psicología, 30(3), $1170-1175$.

Fuente, A., y Herrero-Olaizola, J. (2012). Social integration of Latin-American immigrants in Spain:The influence of the community context. The Spanish Journal of Psychology, 15, 1201-1209. 
García-Cueto, E., Rodríguez-Díaz, F.J., BringasMolleda, C., López-Cepero, J., y Paíno-Quesada, S. (2015). Development of the Gender Role Attitudes Scale (GRAS) amongst young Spanish people. International Journal of Clinical and Health Psychology, 15, 61-68. http://dx.doi.org/10.1016/i.iichp.2014.10.004.

González-Guarda, R.M., Cummings, A.M., Pino, K., y Malhotra, K. (2014). Perceptions of adolescents, parents, and school personnel from a predominantly CubanAmerican community regarding dating and teen dating violence prevention. Research in Nursing and Health, 37, $117-127$.

Herrero, J., Torres, A., Fernández-Suárez, A., y RodríguezDíaz, F.J. (2016). Generalists versus specialists: Toward a typology of batterers in prison. European Journal of Psychology Applied to Legal Context, 8(1), 19-26. http://dx.doi.org/10.1016/i.ejpal.2015.09.002

Ibañez, M.I., Orted, G., Moro, M., Avila, M.I., y Parcet, M.A. (1999).Versión reducida del Cuestionario Revisado de Personalidad de Eysenck (EPQ-RA). Análisis y Modificación de Conducta, 25(104), 849863.

Kline, R.B. (2011). Principles and practice of structural equation modelling (2a.ed.). New York: Guilford.

Koh, K.H., y Zumbo, B. (2008).Multi-group confirmatory factor analysis for testing measurement invariance in mixed item format data. Journal of Modern Applied Statistical Methods, 7(2), 471-477.

López-Cepero, J., Rodríguez-Franco, L., RodríguezDíaz, F.J., y Bringas, C. (2013). Validación de la versión corta del Social Roles Questionnaire (SRQ-R) con una muestra adolescente y juvenil española. Revista Electrónica de Metodología Aplicada, 18, $1-16$

López-Cepero Borrego, J., Rodríguez-Franco, L., Rodríguez-Díaz, F.J., Bringas, C., y Paíno, S.G. (2015). Percepción de la victimización en el noviazgo de adolescentes y jóvenes españoles. Revista Iberoamericana de Psicología y Salud, 6(2), 12-19. http://dx.doi.org/10.1016/i.rips.2015.04.001

Lorenzo-Seva, U., y Ferrando, P.J. (2006). FACTOR: A computer program to fit the exploratory factor analysis model. Behavior Research Methods, Instruments and Computers, 38, 88-91. http://dx.doi.org/10.3758/ BF03192753

Martin, C.E., Houston, A.M., Mmari, K.N., y Decker, M.R. (2012). Urban teens and young adults describe drama, disrespect, dating violence and help-seeking preferences. Maternal and Child Health Journal, 16(5), 957-966.

Mapayi Boladale, M., Yetunde, O., Adesanmi, A., Aloba Olutayo, A., y Olanrewaju, I. (2015).
Personality profiles and psychopathology among students exposed to dating violence at the Obafemi Awolowo University, Ile-Ife. Journal of Interpersonal Violence, 30(1), 168-190.

http://dx.doi.org/10.1177/0886260514532718

Marco, M., Gracia, E., Tomás, J.M., \& López-Quílez, A. (2015). Assessingn eigh borhood disorder: Validation of a three-factor observational scale. European Journal of Psychology Applied to Legal Context, 7(2), 81-89. http://dx.doi.org/10.1016/i.ejpal.2015.05.001

Muthén, B., y Asparouhov, T. (2002). Latent variable analysis with categorical outcomes: Multiple-group and growth modeling in Mplus. Mplus Web Notes: No. 4. www.statmodel.com

Muthén, L. K., y Muthén, B. (2009). Exploratory factor analysis, confirmatory factor analysis and structural equation modeling for continuous outcomes. Recuperado de http://www.statmodel.com/download/ Topic\%201.pdf

Muthén, L.K., y Muthén, B.O. (2010). Mplus User's Guide.Sixth Edition. Los Angeles, CA: Muthénand Muthén. Recuperado de http://www.statmodel.com/ download/usersguide/Mplus\%20Users\%20Guide\%20 v6.pdf

Novo, M., Herbón, J., y Amado, B. (2016). Género y victimización: Efectos en la evaluación de la violencia psicológica sutil y manifiesta, apego adulto y tácticas de resolución de conflictos. Revista Iberoamericana de Psicología y Salud, 7, 89-97.

O’Neil, J.M., Helms, B.J., Gable, R.K., David, L., y Wrightsman, L.S. (1986). Gender-role conflict scale: College men's fear of feminity. Sex Roles, 14, 335-350.

Payne, D.L., Lonsway, K.A., y Fitzgerald, L.F. (1999). Rape myth acceptance: exploration of its structure and its measurement using the Illinois Rape Myth Acceptance Scale. Journal of Research in Personality, 33, 27-68.

Rodríguez, L., Antuña, M.A., López-Cepero, J., Rodríguez, F.J., y Bringas, C. (2012). Tolerance to wards dating violence in Spanish adolescents. Psicothema, 24, 236-242.

Rodríguez-Franco, L., López-Cepero, J., RodríguezDíaz, F.J., Bringas, C., Antuña, A., y Estrada, C. (2010). Validación del cuestionario de violencia entre novios (CUVINO) en jóvenes hispanohablantes: Análisis de resultados en España, México y Argentina. Anuario de Psicología Clínica y de la Salud, 6, 45-52.

Ruiz-Hernández,J.A., García-Jiménez, J.J., LlorEsteban, B., y Godoy-Fernández, C. (2015). Risk factors for intimate partner violence in prison inmates. European Journal of Psychology Applied to Legal Context, 7, 41-49. 
Rueda, H.A., Williams, L.R., y Nagoshi, J.L. (2015). Help-seeking and help-offering for teen dating violence among acculturating Mexican American adolescents. Children and Youth Services Review, 53, 219-228. http://dx.doi.org/10.1016/i.childyouth.2015.04.001

Shorey, R.C., Seavey, A.E., Brasfield, H., Febres, J., Fite, P.J., y Stuart, G.L. (2015). The moderating effect of social support from a dating partner on the association between dating violence victimization and adjustment. Violence against Women, 27(4), 460-477. http://dx.doi.org/10.1177/1077801215570482

Stepteau-Watson, D. (2014). Dating violence, young African American males, and risk and protective factors:A review of the literature. Journal of Human Behavior in the Social Environment, 24, 694-701. http://dx.doi.org/10.1080/10911359.2014.922818

Temple, J.R., Shorey, R.C., Tortolero, S.R., Wolfe, D.A., y Stuart, G.L. (2013). Importance of gender and attitudes about violence in the relationship between exposure to interparental violence and the perpetration of teen dating violence. Child Abuse and Neglect, 37(5), 343-352. http://dx.doi.org/10.1016/i. chiabu.2013.02.001

Timmerman, M.E., y Lorenzo-Seva, U. (2011). Dimensionality assessment of ordered polytomous items with parallel analysis. Psychological Methods, $16,209-220$.
Ulloa, E., Jaycox, L., Skinner, S., y Orsburn, M. (2008). Attitudes about violence and dating among Latino/a boys and girls.Journal of Ethnic and Cultural Diversity in Social Work, 17, 157-176.

Ureña, J., Romero, E.M., Casas, J.A., Viejo, C., y Ortega-Ruiz, R. (2015). Psychometrics properties of Psychological Dating Violence Questionnaire: A study with young couples. International Journal of Clinical and Health Psychology, 15(1), 52-60. http://dx.doi. org/10.1016/i.ijchp.2014.07.002

Vega-Gea, E., Ortega-Ruiz, R., y Sánchez, V. (2016). Peer sexual harassment in adolescence: Dimensions of the sexual harassment survey in boys and girls. International Journal of Clinical and Health Psychology, 16(1), 47-57. http://dx.doi.org/10.1016/i. iichp.2015.08.002

Yu, R., Geddes, J.R., y Fazel, S. (2012). Personality disorders, violence, and antisocial behavior: A systematic review and meta-regression analysis. Journal of Personality Disorders, 26(5), 775-792. 